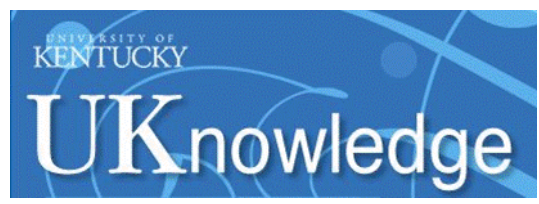

University of Kentucky

UKnowledge

Mechanical Engineering Faculty Publications

Mechanical Engineering

6-2012

\title{
Multi-Dimensional Modeling of Charring Ablators
}

\author{
Haoyue Weng \\ University of Kentucky, kimomt@gmail.com \\ Huaibao Zhang \\ University of Kentucky, paulhuaizhang@uky.edu \\ Ovais U. Khan \\ University of Kentucky \\ Alexandre Martin \\ University of Kentucky, alexandre.martin@uky.edu
}

Follow this and additional works at: https://uknowledge.uky.edu/me_facpub

Part of the Computer Sciences Commons, and the Space Vehicles Commons

Right click to open a feedback form in a new tab to let us know how this document benefits you.

\section{Repository Citation}

Weng, Haoyue; Zhang, Huaibao; Khan, Ovais U.; and Martin, Alexandre, "Multi-Dimensional Modeling of Charring Ablators" (2012). Mechanical Engineering Faculty Publications. 18.

https://uknowledge.uky.edu/me_facpub/18

This Conference Proceeding is brought to you for free and open access by the Mechanical Engineering at UKnowledge. It has been accepted for inclusion in Mechanical Engineering Faculty Publications by an authorized administrator of UKnowledge. For more information, please contact UKnowledge@lsv.uky.edu. 


\section{Multi-Dimensional Modeling of Charring Ablators}

Digital Object Identifier (DOI)

http://dx.doi.org/10.2514/6.2012-2748

\section{Notes/Citation Information}

Published in the Proceedings of the 43rd AIAA Thermophysics Conference, Paper 2012-2748, p. 1-13.

Copyright $\odot 2012$ by Haoyue Weng, Huaibao Zhang, Ovais U. Khan, and Alexandre Martin.

The copyright holders have granted the permission for posting the article here. 


\title{
Multi-dimensional modeling of charring ablators
}

\author{
Haoyue Weng*, Huaibao Zhang Ovais U. Khan $^{\ddagger}$ and Alexandre Martin ${ }^{\S}$ \\ Department of Mechanical Engineering, University of Kentucky, Lexington, KY, 40506, USA
}

\begin{abstract}
Re-entry of a spacecraft occurs at the hypersonic regime where the flow field is extremely complex: high temperature gradients occurring in the shock-layer region ionize and dissociate the air. Even if a large portion of heat generated during this process is convected away in the surrounding air, a fraction of it is still transferred to the vehicle. Therefore, it is important to protect the vehicle with a suitable kind of shielding. Of the many techniques available today, use of ablative material is gaining popularity. The basic idea behind an ablating heat shield is that the energy incident on the spacecraft is used to vaporized the material, thus preventing a significant part of the heat to be transferred into the structure. The available literature indicates that most of the past investigations either do not consider the actual physical processes taking place during ablation, or are limited to a one-dimensional model. The present investigation shows the development of a numerical model for simulating the multi-dimensional heat transfer phenomena that occurred in a typical ablative TPS. The newly developed model is verified using closed form analytical solutions and validated with available data. This effort consists of the first steps of an ongoing project to develop a comprehensive multi-scale, multi-physics and multi-dimensional material response code aimed at modeling charring and surface ablators.
\end{abstract}

\section{Introduction}

TMOspheric entry/re-entry of vehicles takes place in the hypersonic flow regime. In this type of flow, A fluid properties such as pressure, temperature, and density change abruptly as they cross the bow shock, and causes the flow field between the shock and the vehicle surface to become extremely hot. This leads to large temperature gradients and skin friction within the boundary layer, and results in high heat transfer rates to the vehicle surface. Efficient design of the Thermal Protection System (TPS) is necessary in order to reduce the heat conducted into the body surface, optimize the heat shield, and avoid structural failure of the vehicle. One of the available techniques is to use ablative material, which has been proven as an efficient way to reduce the rate of heat transfer into the vehicle. However, ablation modeling is a challenging task as it involves simulating complex processes of mass loss and energy absorption through complex chemical reactions. Moreover, in the case of decomposing (charring) ablators, inner pyrolysis takes place. This results in the formation of porous material as well as inner gas, further increasing the complexity of the numerical model. Several studies have been performed to model the complex thermochemical phenomenon occurring in ablative material for a re-entry vehicle. Ref. 1-3 used control volume finite element method (CVFEM) to model one- and two-dimensional ablation problems. A research group at the University of Michigan has contributed in predicting the design of an efficient thermal protection system. ${ }^{4-7}$ They utilized finite volume based code LeMANS ${ }^{8}$ for evaluating the hypersonic flow field over a vehicle and control volume finite element based code MOPAR for predicting the material response. Their coupling algorithm is based on the method described in Ref. 9. Ref. 10 reevaluated the material response of the Pioneer-Venus vehicles. They made a comparison of thermocouple temperature values with the flight data and found a good agreement between flight data and calculations for the day probe and north probe. However, for the night probe and large probe, thermocouple temperatures were significantly higher than the flight data values. In Ref. 11,12, a one-dimensional modeling of a typical thermal ablation problem considering pyrolysis gas flow through pores

\footnotetext{
* Graduate Research Assistant, AIAA Student Member

$\dagger$ Graduate Research Assistant, AIAA Student Member

$\ddagger_{\text {Research Associate, AIAA Member }}$

$\S$ Assistant Professor, AIAA Senior Member
} 
was performed. A CVFEM was utilized to discretize the coupled governing equations of energy and gas flow through a porous medium. Recently, Ref. 13 presented an implicit system solver to model three-dimensional heat and mass transfer for a charring ablator thermal protection system. Verification of the proposed solver was performed using exact solutions and the Method of Manufactured Solutions for transient heat conduction and porous gas flow problems. Available literature indicates that most of the past investigations either do not consider the actual physical processes occurring during ablation, or are limited to one- and two-dimensional models. In the present investigation an attempt has been made to simulate the actual physics of thermal ablation phenomenon by considering anisotropic, multilayer, variable material properties modeled in threedimensions. The newly developed model is verified using closed form analytical solutions and validated with the available data. This effort consists of the first steps of an ongoing project to develop a comprehensive multi-scale, multi-physics and multi-dimensional material response code aimed at modeling charring and surface ablators. Results show the successful development of an ongoing project for the numerical solution of multi-dimensional heat transfer phenomena that occurred in a typical ablative TPS.

\section{Proposed model}

The current material response solver utilizes FreeCFD ${ }^{14}$ as a general computing framework. FreeCFD is an open source Computational Fluid Dynamic (CFD) solver that enjoys many features. It uses the popular CGNS format for the computational grid and takes advantage of parallel computing through domain decomposition (ParMETIS) and MPI. Also, it is linked to PETSc library, which provides efficient methods for solving large and sparse linear systems. The code is based on a second order finite volume approach and uses a fully implicit first-order backward Euler method for time integration.

The governing equation solved in FreeCFD takes the form of:

$$
\frac{\partial \mathbf{Q}}{\partial t}+\nabla \cdot\left(\mathbf{F}-\mathbf{F}_{\mathbf{d}}\right)=\mathbf{S}
$$

and, the time-implicit control volume approach takes the form of:

$$
\left[\frac{V}{\Delta t} \frac{\partial \mathbf{Q}}{\partial \mathbf{P}}-\frac{\partial \mathbf{R}}{\partial \mathbf{P}}\right] \Delta \mathbf{P}=\mathbf{R}
$$

In this equation, $V$ is the volume of the cell, $\Delta t$ the time step, $\mathbf{Q}$ is the conservative variables vector and $\mathbf{P}$ is the primitive variables vector. The right side of the equation, represented by $\mathbf{R}$, is defined as:

$$
\sum_{\text {faces }}-\mathbf{F}_{\mathbf{n}} A+\mathbf{S} V
$$

where $A$ is the area of each face of the cell, and $\mathbf{F}_{\mathbf{n}}$ is the normal component of the $\mathbf{F}-\mathbf{F}_{\mathbf{d}}$ flux across that face. The primitive formulation on the lefthand side of the equations allows the direct update of the physical variables, and therefore computes them while preserving a conservative formulation.

\section{A. Governing Fluid Dynamics Equations}

Within the numerical context described above, the vectors of conserved variables, primitive variables and source terms respectively take the form of:

$$
\mathbf{Q}=\left(\begin{array}{c}
\rho_{i} \\
\cdots \\
\rho_{N} \\
\rho u \\
\rho v \\
\rho w \\
E
\end{array}\right), \quad \mathbf{P}=\left(\begin{array}{c}
p_{i} \\
\ldots \\
p_{N} \\
u \\
v \\
w \\
T
\end{array}\right), \quad \mathbf{S}=\left(\begin{array}{c}
\dot{w}_{i} \\
\ldots \\
\dot{w}_{N} \\
0 \\
0 \\
0 \\
0
\end{array}\right)
$$

In these expressions, $\rho$ is the total density, $u, v, w$ are the bulk velocity components and $E$ is the total energy per unit volume. The finite-rate chemistry source term is represented by $\dot{w}_{i}$. The inviscid and diffusive flux 
matrices are given by:

$$
\mathbf{F}=\left(\begin{array}{ccc}
\rho_{i} u & \rho_{i} v & \rho_{i} w \\
\ldots & \ldots & \ldots \\
\rho_{N} u & \rho_{N} v & \rho_{N} w \\
\rho u^{2}+p & \rho v u & \rho w u \\
\rho u v & \rho v^{2}+p & \rho w v \\
\rho u w & \rho v w & \rho w^{2}+p \\
(E+p) u & (E+p) v & (E+p) w
\end{array}\right), \quad \quad \mathbf{F}_{\mathbf{d}}=\left(\begin{array}{c}
-\mathbf{J}_{i} \\
\ldots \\
-\mathbf{J}_{N} \\
\\
\tau \\
\tau \mathbf{u}-\mathbf{q}-\sum\left(\mathbf{J}_{i} h_{i}\right)
\end{array}\right)
$$

where $p$ is the pressure, $\boldsymbol{\tau}$ are the viscous tensor, $\mathbf{J}_{i}$ the diffusion flux species $i$, and $\mathbf{q}$ is the directional heat flux vector.

\section{B. Governing Equations for Material Response Code}

The governing equations for the proposed material response model includes mass, momentum and energy conservation for solid and gas medium. Using the formulation described earlier in Eq. 1, the conservative variables, primitive variables, and source terms are:

$$
\mathbf{Q}=\left(\begin{array}{c}
\phi \rho_{g} \\
\rho_{s 1} \\
\vdots \\
\rho_{s_{n s}} \\
\phi \rho_{g} u \\
\phi \rho_{g} v \\
\phi \rho_{g} w \\
\phi E_{g}+E_{s}
\end{array}\right), \quad \mathbf{P}=\left(\begin{array}{c}
\phi \rho_{g} \\
\rho_{s i} \\
\vdots \\
\rho_{s_{n s}} \\
u \\
v \\
w \\
T
\end{array}\right), \quad \mathbf{S}=\left(\begin{array}{c}
\omega_{g} \\
\omega_{s 1} \\
\vdots \\
\omega_{s n s} \\
-\phi \frac{\mu}{K} u \\
-\phi \frac{\mu}{K} v \\
-\phi \frac{\mu}{K} w \\
0
\end{array}\right) .
$$

Here, subscript $g$ and $s$ represent the gas and solid phase, respectively. $\phi$ is the porosity of the material, $\mu$ is the viscosity and $K$ is the permeability. For now, the solid material in the charring ablator is modeled as a porous media, for which the flow through it is governed by Darcy's law. Non-Darcian behavior ${ }^{15}$ will be considered in the future. Note that Darcy's law is integrated in the momentum equations, as shown in vector $\mathbf{S}$.

The convective and diffusive fluxes are given by:

$$
\mathbf{F}=\left(\begin{array}{ccc}
\phi \rho_{g} u & \phi \rho_{g} v & \phi \rho_{g} w \\
0 & 0 & 0 \\
\vdots & \vdots & \vdots \\
0 & 0 & 0 \\
\phi \rho_{g} u^{2}+p & \phi \rho_{g} v u & \phi \rho_{g} w u \\
\phi \rho_{g} u v & \phi \rho_{g} v^{2}+p & \phi \rho_{g} w v \\
\phi \rho_{g} u w & \phi \rho_{g} v w & \phi \rho_{g} w^{2}+p \\
\phi \rho_{g} u H & \phi \rho_{g} v H & \phi \rho_{g} w H
\end{array}\right) \quad, \quad \mathbf{F}_{\mathbf{d}}=\left(\begin{array}{ccc}
0 & 0 & 0 \\
0 & 0 & 0 \\
\vdots & \vdots & \vdots \\
0 & 0 & 0 \\
0 & 0 & 0 \\
0 & 0 & 0 \\
0 & 0 & 0 \\
\multicolumn{2}{c}{\boldsymbol{\kappa} \cdot \boldsymbol{\nabla} T} &
\end{array}\right) .
$$

Where $\boldsymbol{\kappa}$ is the conductivity tensor. As can be seen in the governing equations, the solid material includes multiple species. The total density of the material is computed with:

$$
\rho_{s}=\sum_{i=1}^{n s} \Gamma_{i} \rho_{i},
$$

where $\Gamma_{i}$ is the volume fraction of species $i$ in the virgin composite. The solid decomposition rate of each component can be evaluated by performing a temporal derivative of Eq. 4 :

$$
\frac{\partial \rho_{s}}{\partial t}=\sum_{i=1}^{n s} \Gamma_{i} \frac{\partial \rho_{i}}{\partial t} .
$$


The decomposition of each component is assumed to follow a modified Arrhenius relation given by

$$
\omega_{s i}=\frac{\partial \rho_{i}}{\partial t}=-A_{i} \rho_{v_{i}}\left(\frac{\rho_{s i}-\rho_{c i}}{\rho_{v_{i}}}\right)^{\psi_{i}} \exp \left(\frac{-E_{i}}{R T}\right), \quad T>T_{\text {react }_{i}},
$$

where subscript $v$ and $c$ are respectively for the virgin and charred state of the solid material. The source terms for the solid and mass equations balance themselves, thus ensuring total mass conservation:

$$
\omega_{g}=-\sum_{i=1}^{n s} \Gamma_{i} \omega_{s i}
$$

The above mentioned governing equations have been implemented in the material response code. An example of results using the current code in three dimension is shown in Fig. 1.

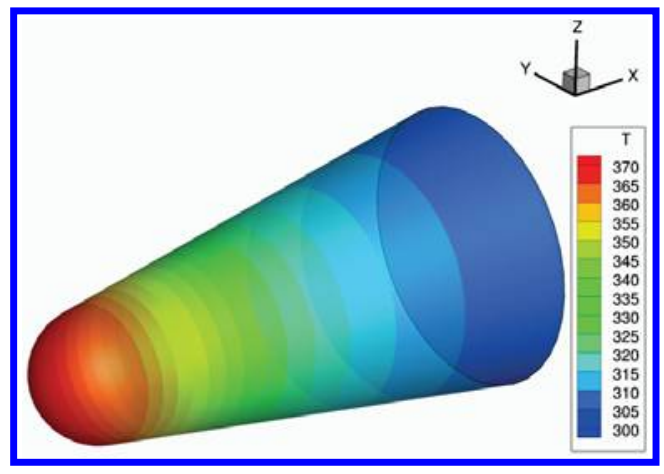

Figure 1. Example of a 3D simulation using the material response code

\section{Results: Fluid Dynamics}

Although freeCFD is designed to compute material and flow field solutions in an integrated manner, the multiple time-scale present in a hypersonic re-entry problem ${ }^{5}$ involves modifications to the treatment of time steps. For the results presented here, solutions are obtained independently for flow field and the material response.

As a first test-case, a 2-D hypersonic Mach 10 flow over a 1 meter radius cylinder is presented. Because of the limitation of the code as far as thermal nonequilibrium is concerned, a simple gas, Argon, is used. The free stream conditions used in the problem are listed in Table 1, and Fig. 2 shows the computational grid. As can be seen, grid clustering at the surface and shock alignment is introduced to capture important effects.

Table 1. Free stream conditions for the flow field test case

\begin{tabular}{ccccc}
\hline \hline Mach Number & Velocity & Density & Temperature & Pressure \\
\hline 10 & $2624.0 \mathrm{~m} / \mathrm{s}$ & $1.408 \times 10^{-4} \mathrm{~kg} / \mathrm{m}^{3}$ & $200.0 \mathrm{~K}$ & $5.8572 \mathrm{~Pa}$ \\
\hline \hline
\end{tabular}

The pressure distribution obtained from the fluid dynamic solver is shown in Fig. 3a). Formation of strong bow shock wave in front of the body is present, and the isocontours are smooth, as expected. Fig. 3b) depicts the corresponding temperature field for the present analysis.

Moreover, pressure and temperature obtained along the stagnation stream line are presented in Fig. 4 and 5. A comparison with the results obtained from CFD code LeMANS, ${ }^{8}$ indicates that the the results are generally in good agreement; however, some deviation near the shock and stagnation regions are observed. These discrepancies are most likely due to a difference in the mesh refinement in the shock region. 


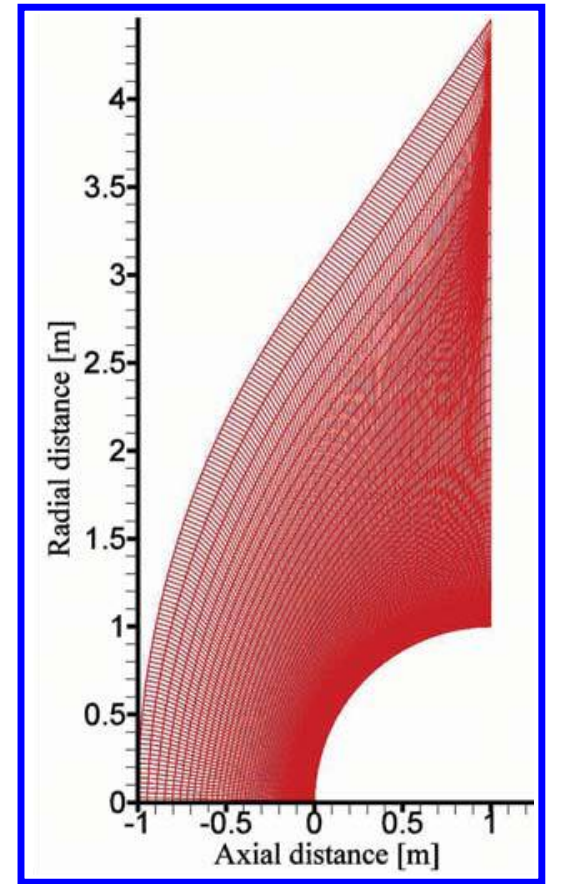

Figure 2. Computational grid used for simulation of Mach 10 Argon flow over cylinder.

\section{Results: Material Response}

The desired outcome of the current work is to be an experimental validation of material response with a 3D geometry, an in-depth comparison of thermal response between ablative and non-ablative materials and an investigation on the behavior of anisotropic effect in a charring ablator. For the preliminary results presented here, a 1-D ablation problem is validated using previous work in the community ${ }^{16}$ and the anisotropic effect in a 3-D non-ablative material is investigated. It is to be noted that the code has been previously verified using most of the test problems presented in Ref. 17.

\section{Material response: 1-D TACOT ablation}

In order to validate the material response model and the numerical scheme, a simple test case is presented: a 0.05 meter TACOT cube with a face kept at a constant temperature of $1644 \mathrm{~K}$, during a minute. The full set of material properties used for the simulation are presented in Ref. 16. Although this is a 1-D problem, the test case is solved on a cube. The mesh of the cube is composed of 15,000 cells (600 in the direction of the applied heat flux) with clustering on the face where the heat is applied.

The material response code MOPAR ${ }^{15}$ is used for code-to-code comparison. The results are presented in 1-D with heating applied on the left side. The profile of solid density, temperature, and gas enthalpy are respectively presented in Fig. 6, Fig. 7, and Fig. 8. Despite a small discrepancy of gas enthalpy in Fig. 8 at the trailing edge of the 20 seconds curve, attributed to a different enthalpy of pyrolysis, the rest shows excellent agreement with the reference results.

\section{Material response: IRV-2 test vehicle}

The next test case models the well defined geometry of the IRV-2 vehicle, ${ }^{9}$ which was extensively used in the past for thermal response validations. ${ }^{4,18}$ For this simulation, the carbon-carbon properties (non-charring) defined in Ref. 17 are used. A 2D view projection of the computational grid for the vehicle is shown in Fig 9 , and the test conditions are presented in Table 2 .

Total heat flux has been evaluated using a hypersonic aerothermodynamic CFD code ${ }^{8}$ and is applied at the vehicle surface. Insulated wall boundary conditions are imposed at the remaining boundaries. The heat flux distribution is shown in Fig. 10. 


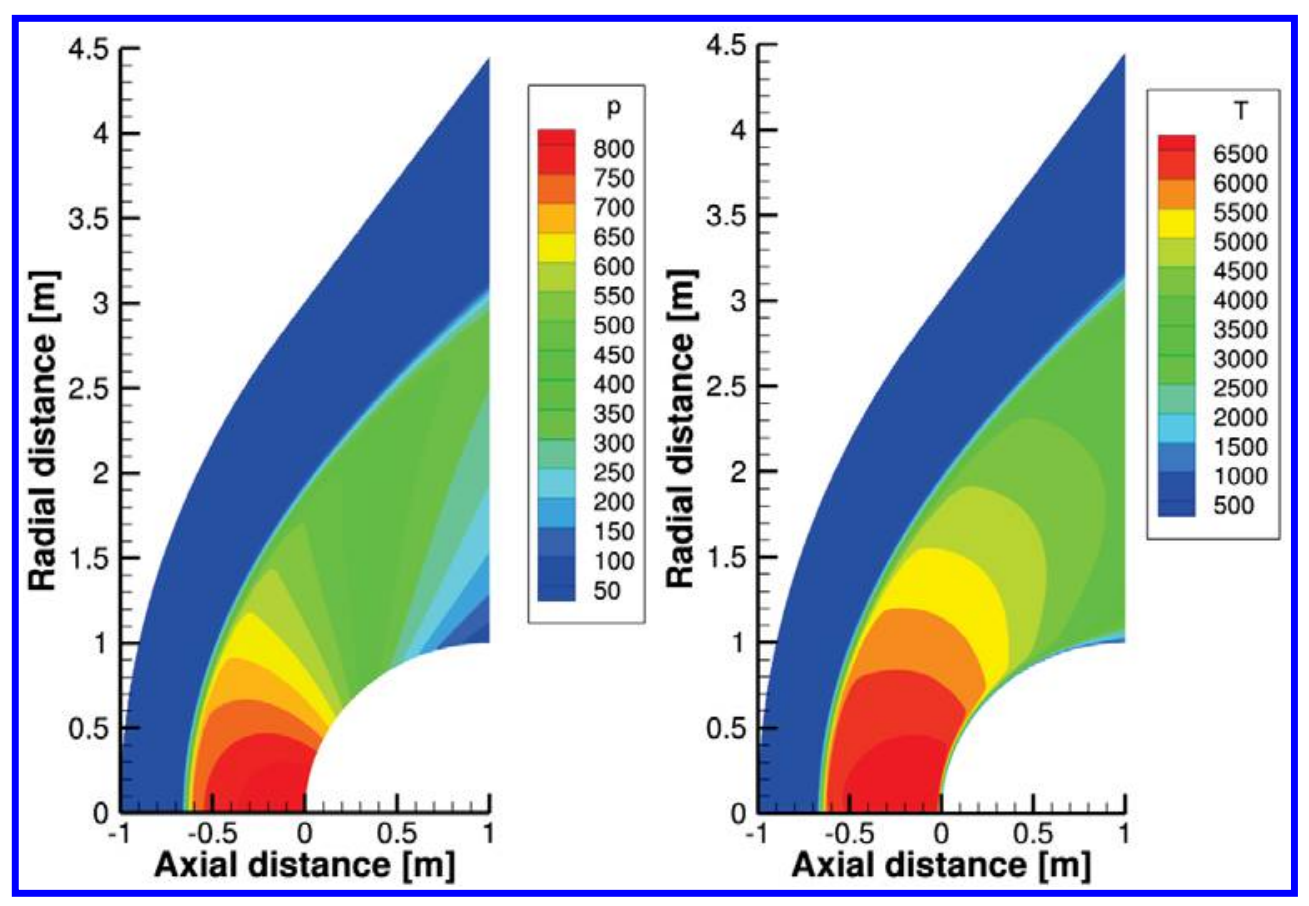

(a) Pressure

(b) Temperature

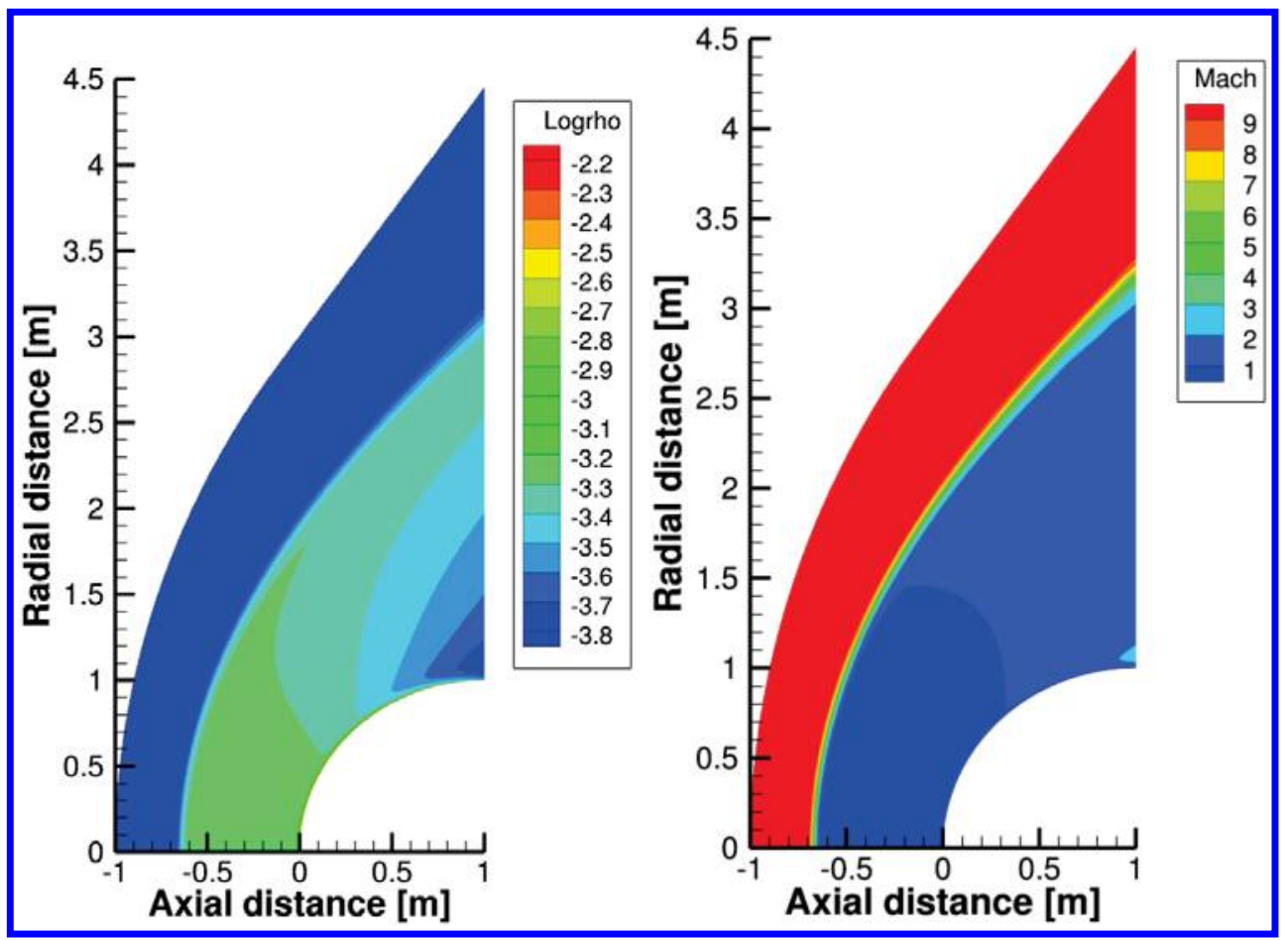

(c) Density

(d) Mach

Figure 3. Isocontours for the Mach 10 Argon flow over cylinder. 


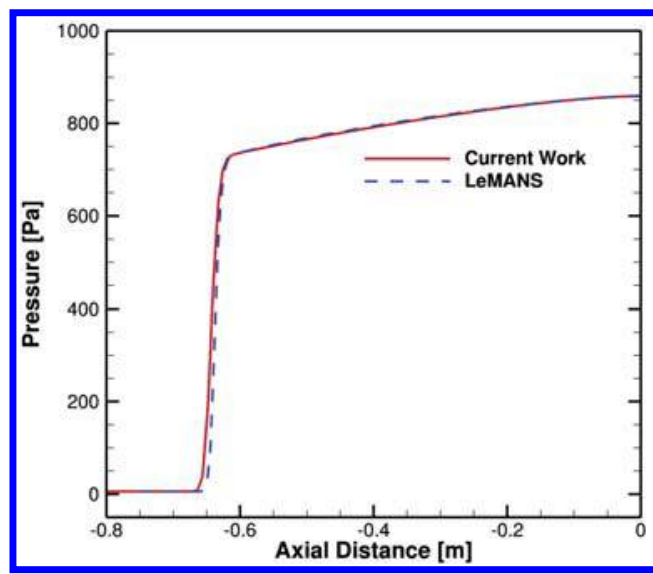

(a) Pressure

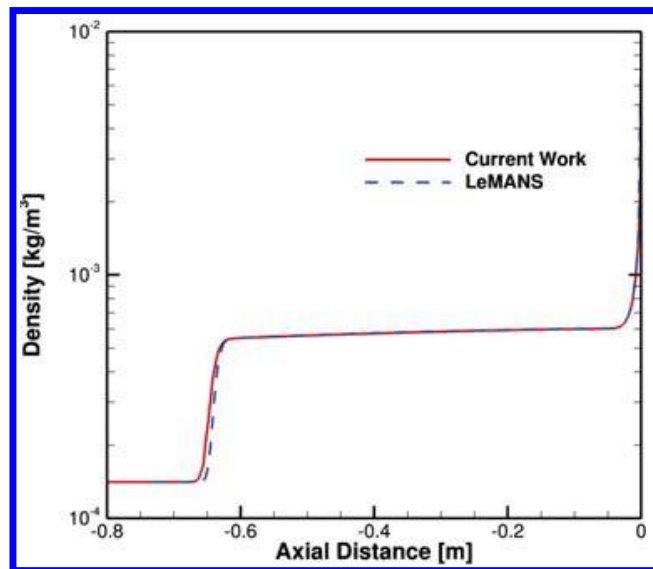

(c) Density

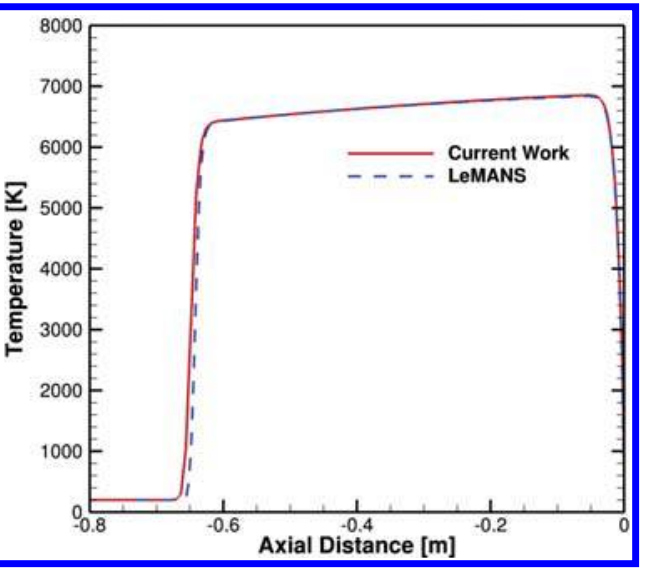

(b) Temperature

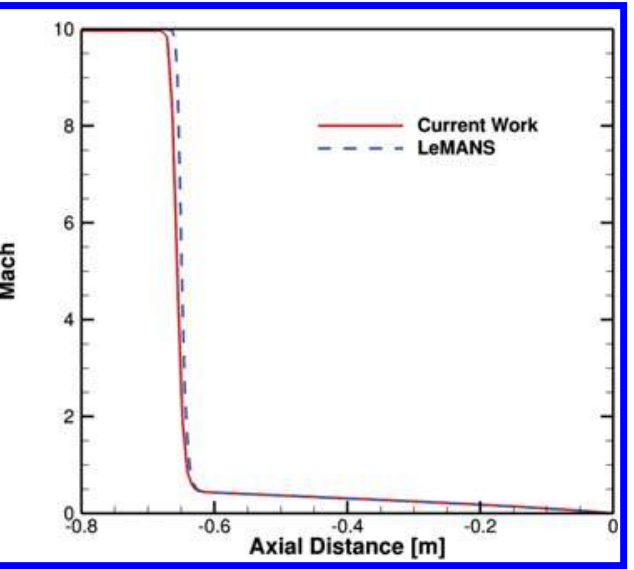

(d) Mach

Figure 4. Stagnation line results for the Mach 10 Argon flow over cylinder.

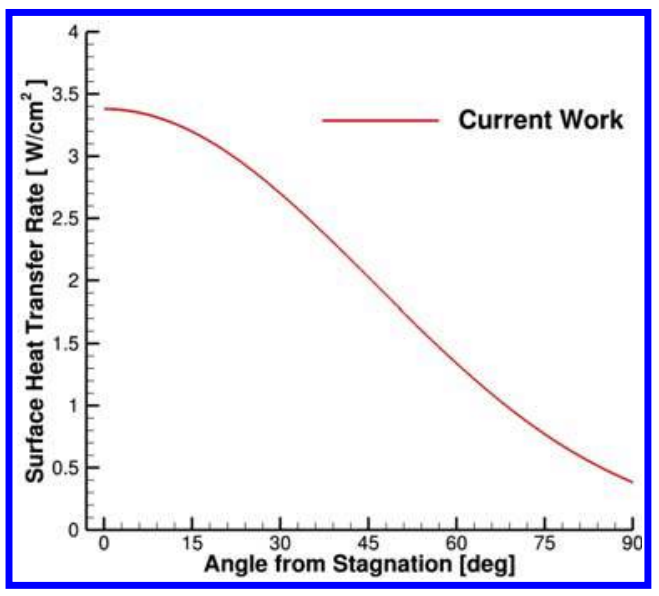

Figure 5. Surface heat flux for the Mach 10 Argon flow over cylinder. 


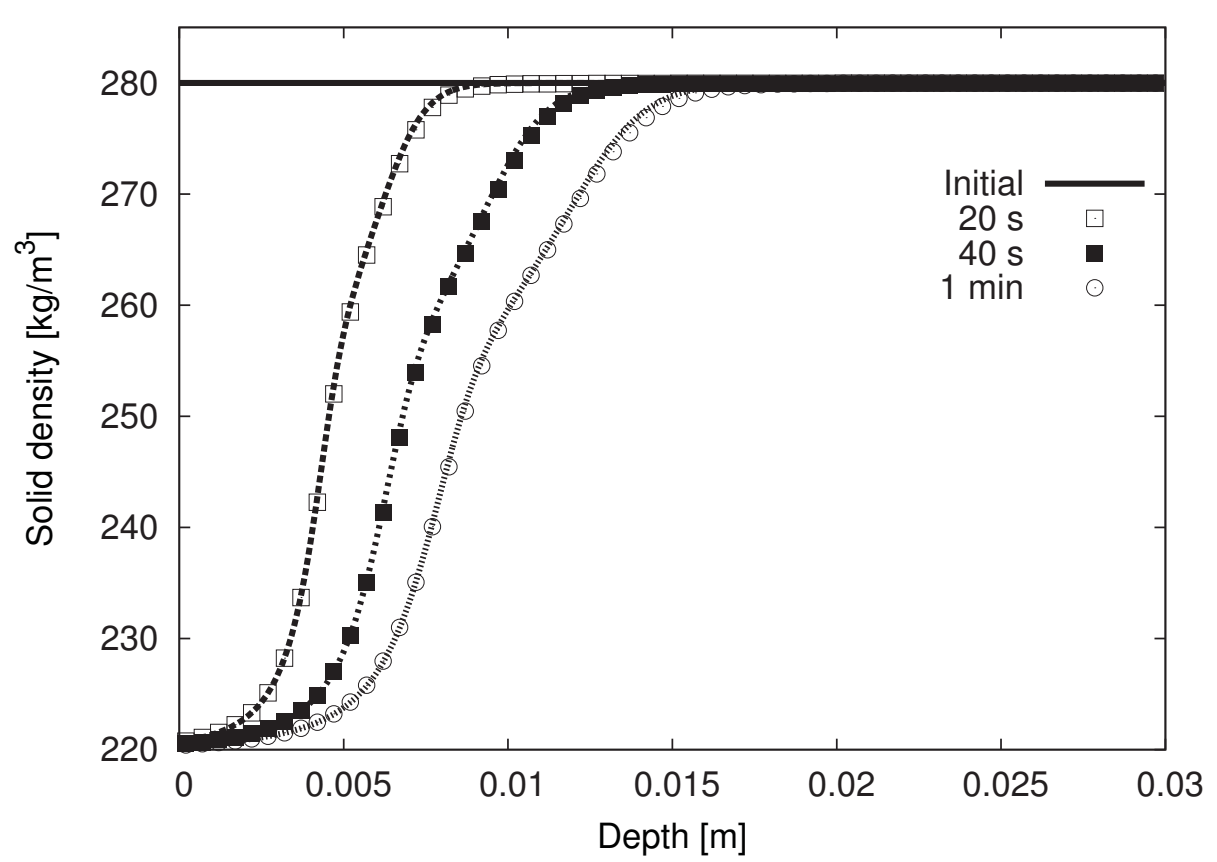

Figure 6. Material density profiles comparison between MOPAR (dash lines) and current work (symbols)

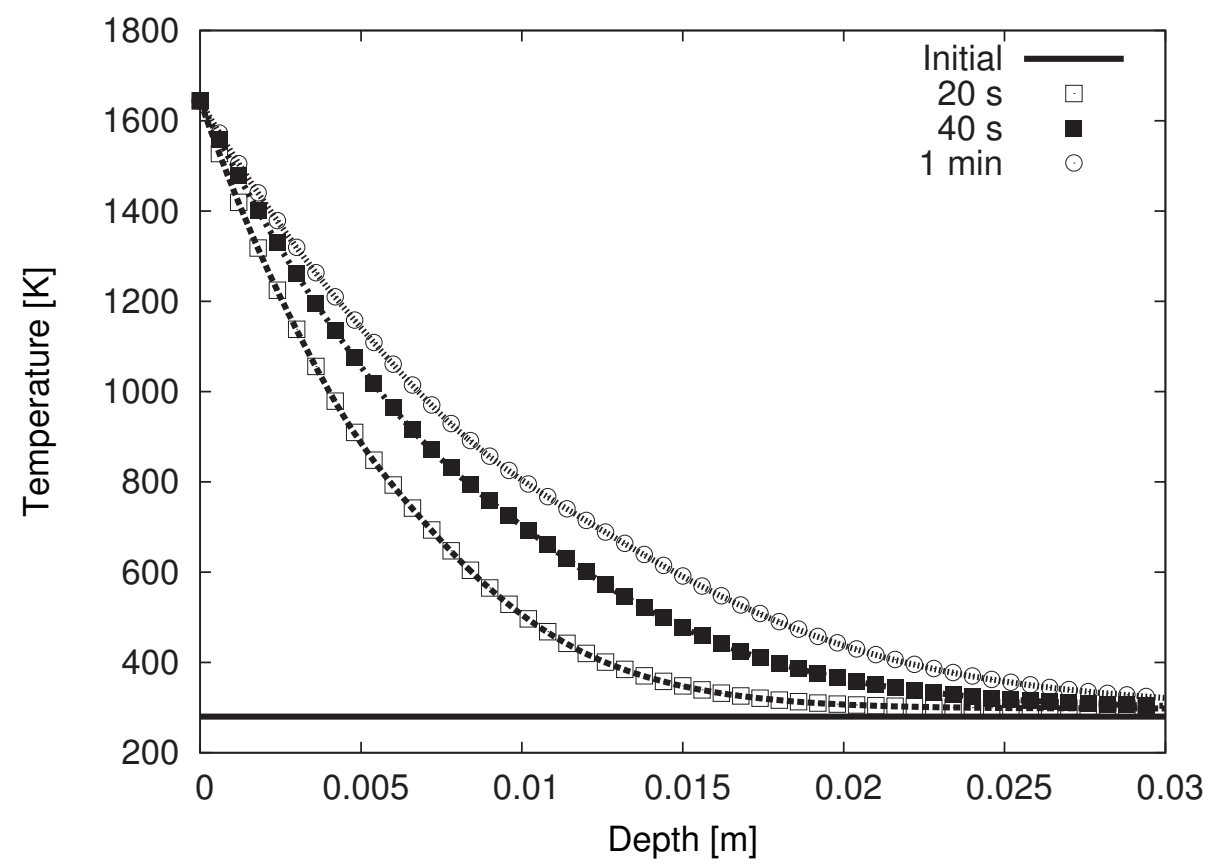

Figure 7. Temperature profiles comparison between MOPAR (dash lines) and current work (symbols) 


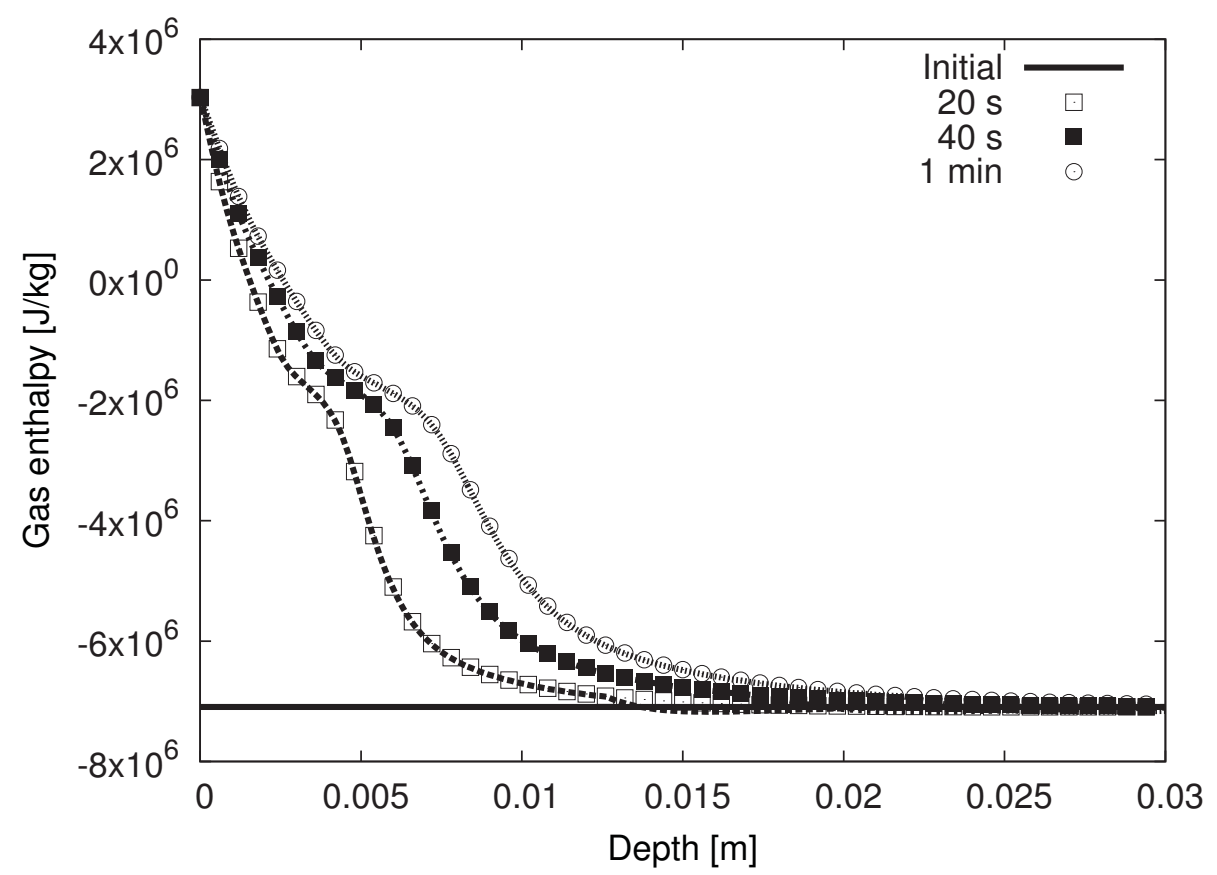

Figure 8. Gas enthalpy profiles comparison between MOPAR (dash lines) and current work (symbols)

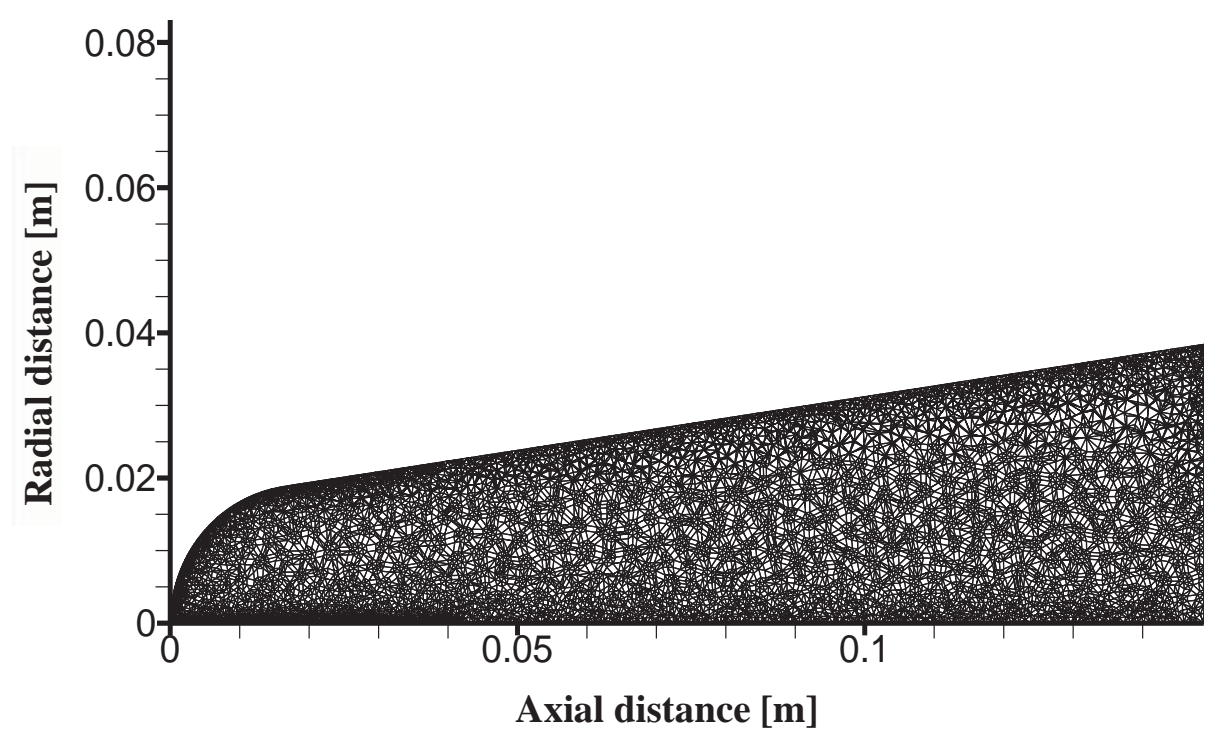

Figure 9. Computational grid for IRV-2 vehicle.

Table 2. Initial condition for the material response test case

\begin{tabular}{ccc}
\hline \hline Temperature & Density & Heat flux \\
\hline $220.0 \mathrm{~K}$ & $1878.96 \mathrm{~kg} / \mathrm{m}^{3}$ & See Fig. 9 \\
\hline \hline
\end{tabular}




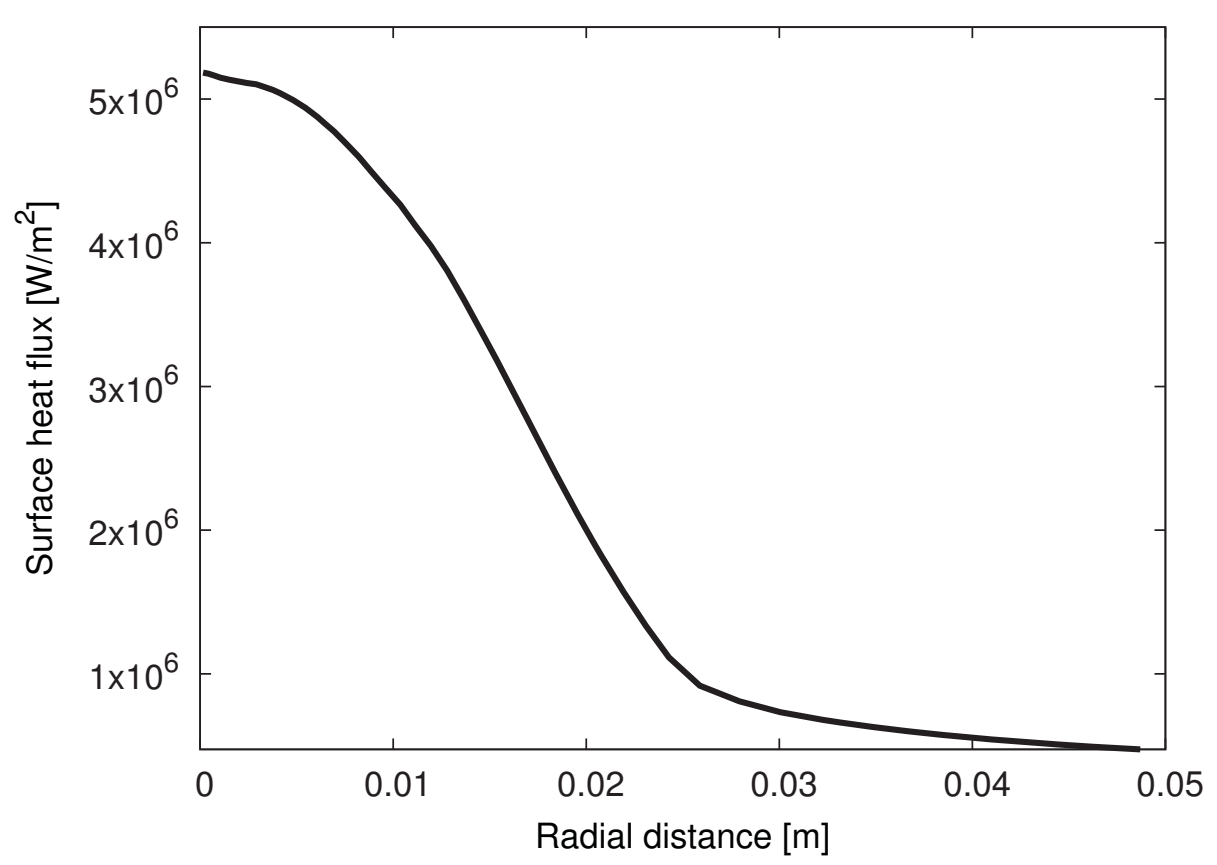

Figure 10. Surface heat flux over IRV-2 vehicle.

Figure 11a) illustrates the computed temperature distribution for the IRV-2 vehicle after 4 seconds of constant exposure. As expected, maximum levels of temperature is observed at the blunted nose section of the body. A gradual decrease in temperature values are also apparent as one move towards the base section. Figure 11b) to c) illustrates the same results while using different values of an anisotropic thermal conductivity. These results are not physical since the off-diagonal coefficients of the conductivity tensor are not consider. However, as can be seen, the results are significantly different from one configuration to another.

\section{Conclusion}

This work shows the initial step of the development of an integrated coupled fluid-material solver for modeling anisotropic and multi-dimensional charring ablators. The solver, part of an integrated CFD framework that allows the simulation of hypersonic flow, models the complex physical processes that occur during a typical atmospheric re-entry. The fluid part of the code has demonstrated the capability of solving hypersonic re-entry problems, and compares well with another established code. A series of validation test-cases have been presented for the material response part, and results of anisotropic thermal conductivity behavior have been presented using the well established IRV-2 test-case. These results shows that the material response code is an efficient and robust method to solve ablation problems in three-dimensional geometry.

\section{Acknowledgments}

Financial support for this work was provided by NASA SBIR Phase-2 Award NNX10CC53P, and NASA Kentucky EPSCoR Award NNX10AV39A. The authors would like to thank Dr. Emre Sozer at NASA Ames for several useful discussions, as well as Hicham Alkandry and Iain D. Boyd, from the University of Michigan, for providing the LeMANS results. 


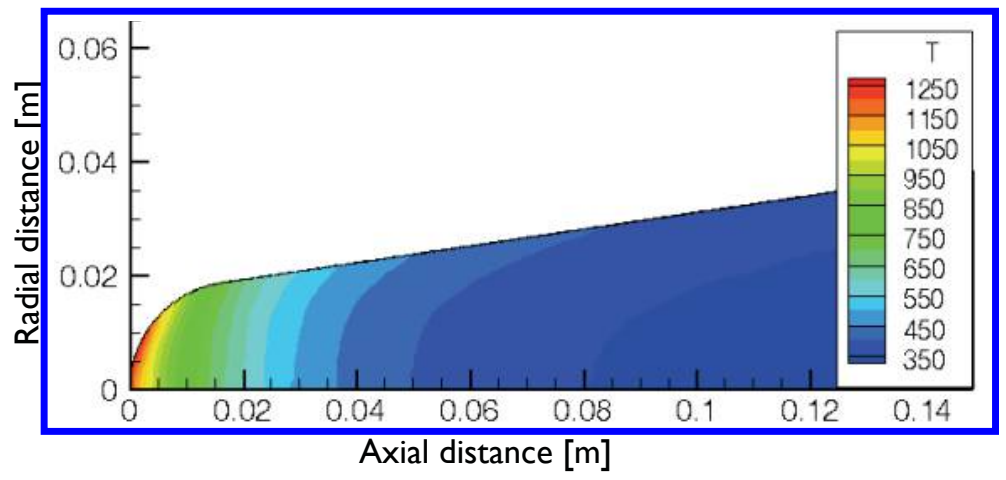

(a) Isotropic thermal conductivity of $k_{x x}=k_{y y}=50$

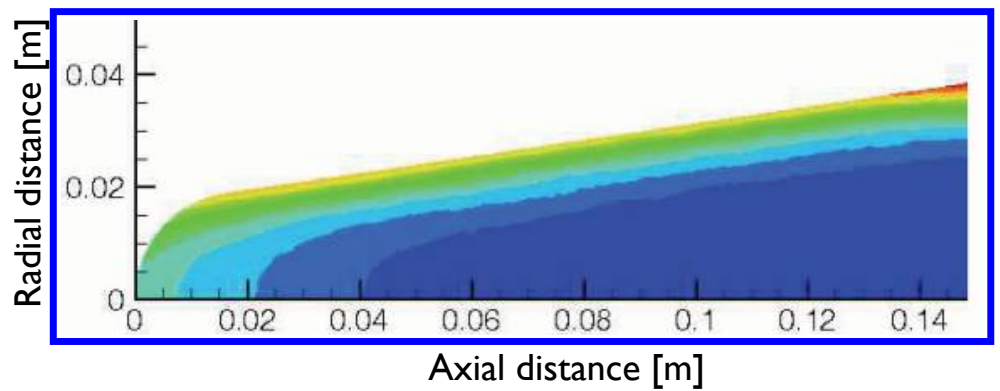

(b) Anisotropic Thermal conductivity of $k_{x x}=5$ and $k_{y y}=50$

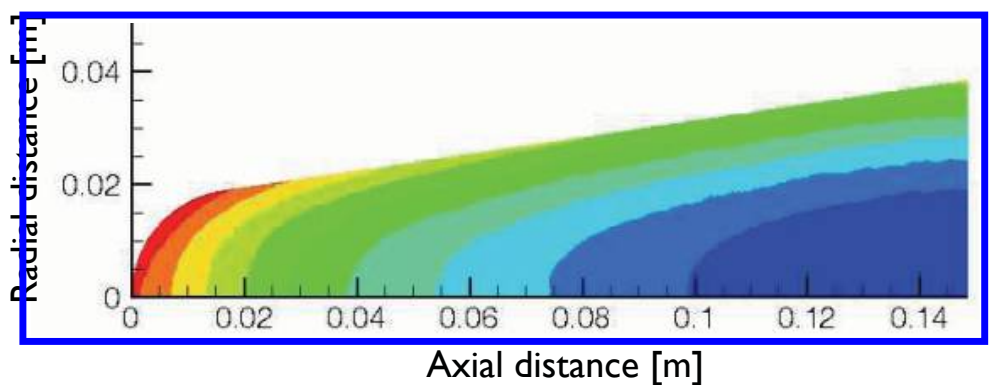

(c) Anisotropic Thermal conductivity of $k_{x x}=50$ and $k_{y y}=5$

Figure 11. Temperature distribution within the IRV-2 vehicle after 4 seconds of exposure. On the figure, the $\mathrm{x}$-axis is aligned with the axial distance axis and the y-axis, with the radial distance axis. The off-diagonal coefficients in the conductivity tensor $\kappa$ are set to zero. 


\section{References}

${ }^{1}$ Blackwell, B., "Numerical prediction of one-dimensional ablation using a finite control volume procedure with exponential differencing," Numerical Heat Transfer, Vol. 14, No. 1, 1988, pp. 17-34.

-2Blackwell, B. F. and Hogan, R. E., "One-Dimensional Ablation Using Landau Transformation and Finite Control Volume Procedure," Journal of Thermophysics and Heat Transfer, Vol. 8, No. 2, April-June 1994, pp. 282-287.

${ }^{3}$ Hogan, R. E., Blackwell, B. F., and Cochran, R. J., "Application of Moving Grid Control Volume Finite Element Method to Ablation Problems," Journal of Thermophysics and Heat Transfer, Vol. 10, No. 2, April-June 1996.

- ${ }^{4}$ Martin, A. and Boyd, I. D., "Simulation of pyrolysis gas within a thermal protection system," 40th AIAA Thermophysics Conference, No. AIAA-2008-3805, Seattle, WA, June 23-26 2008.

${ }^{5}$ Martin, A. and Boyd, I. D., "Implicit implementation of material response and moving meshes for hypersonic re-entry ablation," 47th AIAA Aerospace Sciences Meeting and Exhibit, No. AIAA-2009-0670, Orlando, FL, Jan. 5-8 2009.

${ }^{6}$ Martin, A. and Boyd, I. D., "Assessment of carbon-phenolic-in-air chemistry models for atmospheric re-entry," 10th AIAA/ASME Joint Thermophysics and Heat Transfer Conference, No. AIAA-2010-4656, Chicago, IL, June 28th to July 1st 2010.

${ }^{7}$ Martin, A., Boyd, I. D., Cozmuta, I., and Wright, M. J., "Chemistry model for ablating carbon-phenolic material during atmospheric re-entry," 48th AIAA Aerospace Sciences Meeting and Exhibit, No. AIAA-2010-1175, Orlando, FL, January 2010.

${ }^{8}$ Martin, A., Scalabrin, L. C., and Boyd, I. D., "High performance modeling of an atmospheric re-entry vehicle," Journal of Physics: Conference Series, Vol. 341, 2012, pp. 1-13.

$\checkmark{ }^{9}$ Kuntz, D., Hassan, B., and Potter, D., "Predictions of Ablating Hypersonic Vehicles Using an Iterative Coupled Fluid/Thermal Approach," Journal of Thermophysics and Heat Transfer, Vol. 15, No. 2, 2001, pp. 129-139.

${ }^{10}$ Ahn, H.-K., Park, C., and Sawada, K., "Response of Heatshield Material at Stagnation Point of Pioneer-Venus Probes," Journal of Thermophysics and Heat Transfer, Vol. 16, No. 3, July-September 2002, pp. 432-439.

${ }^{11}$ Amar, A. J., Blackwell, B. F., and Edwards, J. R., "One-Dimensional Ablation Using a Full Newton's Method and Finite Control Volume Procedure," Journal of Thermophysics and Heat Transfer, Vol. 22, No. 1, January 2008, pp. 72-82.

12Amar, A. J., Blackwell, B. F., and Edwards, J. R., "Development and Verification of a One-Dimensional Ablation Code Including Pyrolysis Gas Flow," Journal of Thermophysics and Heat Transfer, Vol. 23, No. 1, January-March 2009 , pp. 59-71.

${ }^{13}$ Amar, A. J., Calvert, N. D., and Kirk, B. S., "Development and Verification of the Charring Ablating Thermal Protection Implicit System Solver," 49th AIAA Aerospace Sciences Meeting, No. AIAA-2011-144, Orlando, FL, Jan. 4-7 2011.

${ }^{14}$ Sozer, E. and Trizilla, P., "freeCFD," http://www.freecfd.com, August 2010.

${ }^{15}$ Martin, A. and Boyd, I. D., "Non-Darcian behavior of pyrolysis gas in a thermal protection system," Journal of Thermophysics and Heat Transfer, Vol. 24, No. 1, Jan.-Mar. 2010.

${ }^{16}$ Lachaud, J., Martin, A., Cozmuta, I., and Laub, B., "Ablation test-case series 1," 4th AFOSR/SNL/NASA Ablation Workshop, 2010.

${ }^{17}$ Amar, A. J., Modeling of One-Dimensional Ablation with porous Flow Using Finite Control Volume Procedure, Master's thesis, North Carolina State University, Raleigh, NC, 2006.

${ }^{18}$ Gnoffo, P. A., Johnston, C. O., and Thompson, R. A., "Implementation of Radiation, Ablation, and Free Energy Minimization Modules for Coupled Simulations of Hypersonic Flow," 47th AIAA Aerospace Sciences Meeting and Exhibit, No. AIAA 2009-1399, Orlando, FL, January 5-8 2009, p. 12. 
This article has been cited by:

1. Haoyue Weng, Alexandre Martin. Numerical Investigation of Thermal Response Using Orthotropic Charring Ablative Material. Journal of Thermophysics and Heat Transfer, ahead of print1-10. [Abstract] [Full Text] [PDF] [PDF Plus]

2. Haoyue Weng, Alexandre Martin. 2014. Multidimensional Modeling of Pyrolysis Gas Transport Inside Charring Ablative Materials. Journal of Thermophysics and Heat Transfer 28:4, 583-597. [Abstract] [Full Text] [PDF] [PDF Plus] 